\title{
Change in entropy of the spinning black holes
}

\author{
Dipo Mahto ${ }^{1}$, Amresh Kumar Singh ${ }^{2}$, Kumari Vineeta ${ }^{3}$, Ashok Kumar ${ }^{4}$ \\ ${ }^{1}$ Department of Physics, Marwari College, T. M. B. U. Bhagalpur - 812007, India \\ ${ }^{2}$ University Department of Physics, T. M. B. U. Bhagalpur - 812007, India \\ ${ }^{3}$ Department of Education, S.M. College, T. M. B. U. Bhagalpur - 812007, India \\ ${ }^{4}$ Department of Physics, M.R.J.D. College, Bishnupur, Begusarai - 851101, India \\ ${ }^{1-4}$ E-mail address: dipomahto@hotmail.com , amresh.skc@gmail.com , \\ vineeta.priyadarshi@gmail.com ,drashokbgs259@gmail.com
}

\begin{abstract}
Aims: To derive an expression for change in entropy of spinning black holes on the basis of the model for the energy of spinning black holes $E_{B H s}=K_{B H s} R_{s}^{\prime}$ ( Mahto et al. 2011a) \& the model for entropy change $\delta S_{b h}=\frac{2 \pi}{\kappa \cdot c^{2}} \delta E$ ( Mahto et al. 2011b) and then calculate their values for different test spinning black holes. Study Design: Data for the mass of black holes have collected from the research paper entitled :Super massive Black Holes in Galactic Nuclei: Past Present \& Future Research(2005), Space Science Reviews by L. Ferrarese \& H. Ford and Black holes in Astrophysics(2005), New Journal Physics by R. Narayan. The data for black hole constant for spinning black holes $K_{B H s}=1.214 \times 10^{44} \mathrm{~J} / \mathrm{m}$ ) is taken from the paper entitled: Study of Schwarzschild radius with reference to the spinning black holes. Bulletin of Pure and Applied Sciences (2011a). Place and Duration of Study: Department of Physics, Marwari College Bhagalpur and University Department of Physics, T.M.B.U. Bhagalpur, between December 2013 and March 2014. Methodology: A theoretical based work using Laptop to calculate the calculation for change in entropy of different test spinning black holes at Marwari College Bhagalpur and the residential research chamber of the first author. Results: The calculation shows that the change in entropy of spinning black holes of the rest masses for stellar - mass black holes $\left(\mathrm{M} \sim 5-20 \mathrm{M}_{\odot}\right)$ in X-ray binaries is $2.483 \times 10^{63}$ to $39.731 \times 10^{63} \mathrm{~J} / \mathrm{K}$ and for the super massive black holes $\left(\mathrm{M} \sim 10^{6}-10^{9.5} \mathrm{M}_{\odot}\right)$ in active galactic nuclei is $9.932 \times 10^{73}$ to $28.432 \times 10^{80} \mathrm{~J} / \mathrm{K}$. The nature of the graph for XRBs is the same to the Hawking entropy with the event horizon and straight line for AGN which confirms the validity of equations $E_{B H s}=K_{B H s} R_{s}^{\prime}$ and $\delta S_{b h}=\frac{2 \pi}{\kappa \cdot c^{2}} \delta E$. Conclusion: The change in energy and entropy of black holes are mainly dependent on the mass and independent of their event horizons.
\end{abstract}

Keywords: Event-horizon; AGN; Entropy \& Energy 


\section{INTRODUCTION}

Classically, the black holes are perfect absorbers and do not emit anything; their temperature is absolute zero. However, in quantum theory black holes emit Hawking radiation with a perfect thermal spectrum. This allows a consistent interpretation of the laws of black hole mechanics as physically corresponding to the ordinary laws of thermodynamics [1]. The laws of black hole mechanics as proposed by Bardeen et al. describe the behaviour of a black hole in close analogy to the laws thermodynamics by relating mass to energy, area to entropy, and surface gravity to temperature [2]. Dipo Mahto et al. have derived the formula for the energy of spinning black holes in terms of the radius of the event horizon [3]. Kanak Kumari et al. have derived the formula for the energy of non-spinning black holes in terms of the radius of the event horizon [4]. Dipo Mahto et al. derived an expression for the change in energy and entropy of Non-spinning black holes taking account the first law of the black hole mechanics relating the change in mass $\mathrm{M}$, angular momentum $\mathrm{J}$, horizon area A and charge $\mathrm{Q}$, of a stationary black hole with Einstein's mass-energy equivalence relation [5]. Dipo Mahto et al. derived an expression for change in entropy of non-spinning black holes on the basis of formula $\left(E_{B H}=K_{B H} R_{s}\right.$ ) as proposed by Kanak Kumari et al. (2010) and the formula $\delta S_{b h}=\frac{2 \pi}{\kappa \cdot c^{2}} \delta E$ ( Mahto et al. 2011b) and calculated their values for different test nonspinning black holes [6]. In the present paper, we have derived an expression for change in entropy of spinning black holes using [3] the model $\left(E_{B H s}=K_{B H s} R_{s}^{\prime}\right)$ and the formula $\delta S_{b h}=\frac{2 \pi}{\kappa \cdot c^{2}} \delta E[5]$ and then calculated their values for different test spinning black holes.

\section{DISCUSSION}

The Black hole possesses an event horizon (a one-way membrane) that casually isolates the "inside" of the Black hole from the rest of the universe. The radius of the event horizon of a non-spinning BH given by the Schwarzschild radius in terms of solar mass can be obtained as [7]

$$
R_{s}=2950\left(M / M_{\odot}\right) m
$$

In the case of spinning black holes, the equation can be written as

$$
R_{s}^{\prime}=1475\left(M / M_{\odot}\right) m
$$

The energy of spinning black holes in terms of radius of event horizon is given as [3]

$$
E_{B H s}=K_{B H s} R_{s}^{\prime}
$$

where $K_{B H s}=1.214 \times 10^{44} \mathrm{~J} / \mathrm{m}$

Differentiating eq $^{\mathrm{n}}(3)$, we have

$\delta E_{B H s}=K_{B H s} \delta R_{s}^{\prime}$ 
The change in entropy of different test Non-spinning black holes for their corresponding change in energy is given by the following eqn [5]

$\delta S_{b h}=\frac{2 \pi}{\kappa \cdot c^{2}} \delta E$

Putting eq ${ }^{\mathrm{n}}$ (4) in the above eqn, we have

$\delta S_{b h}=\frac{2 \pi}{\kappa c^{2}} K_{B H s} \delta R_{s}^{\prime}$

Writing $\delta S_{b h s}$ and $\delta R_{b h s}$ instead of $\delta S_{b h}$ and $\delta R_{s}^{\prime}$ stand for the change in entropy and radius of event horizon respectively, we have.

$\delta S_{b h s}=\frac{2 \pi}{\kappa c^{2}} K_{B H s} \delta R_{b h s}$

where $\kappa$ and $R_{b h s}$ are the surface gravity \& radius of the event horizon of spinning black holes given as $[8,10]$

$\kappa=\frac{1}{4 M}$

The term M stands for the mass of black holes. From eqn. (9), it is clear that the surface gravity of black hole is inversely proportional to its mass and the different black holes will have different surface gravity. The role of surface gravity $(\kappa)$ may be seen in the research paper $[8,10]$.

The equation (8) can be used to calculate the change in entropy of different test spinning black holes for their corresponding change in the radius of the event horizon.

\section{DATA IN THE SUPPORT OF MASS OF BLACK HOLES}

There are two categories of black holes classified on the basis of their masses clearly very distinct from each other, with very different masses $\mathrm{M} \sim 5-20 \mathrm{M}_{\odot}$ for stellar - mass black holes in X-ray binaries and $\mathrm{M} \sim 10^{6}-10^{9.5} \mathrm{M}_{\odot}$ for super massive black holes in active galactic nuclei $[10,11]$. The mass of black holes never be greater than $5 \times 10^{9} \mathrm{M}_{\odot}$ [17]. The other data in the support of mass of black holes in AGN can seen in references [11-16].

On the basis of the data mentioned above regarding the mass of black holes in XRBs and AGN in terms of solar masses, we have calculated change in entropy of spinning black holes in XRBs and AGN for given radius of event horizon of different test spinning black holes listed in the Table $1 \& 2$ respectively.

There is negligible change in the radius of the event horizon due to change in entropy. Hence in the numerical calculation, the radius of the event horizon $\left(R_{b h s}\right)$ can be used instead of $\delta R_{b h s}[5]$. 
Table 1.

\begin{tabular}{|c|c|c|c|}
\hline \multicolumn{4}{|c|}{ Change in entropy of spinning black holes in XRBs } \\
\hline Sl. No & $\begin{array}{l}\text { Mass of } \\
\text { black } \\
\text { holes } \\
\text { (M) }\end{array}$ & $\begin{array}{l}\text { Radius of the event } \\
\quad \text { horizon } \\
R_{b h s}=1475\left(M / M_{\odot}\right) \\
\quad \text { (in metre) }\end{array}$ & $\begin{array}{c}\text { Entropy change of black } \\
\text { holes }\left(\delta S_{b h}\right) \text { in } \mathrm{J} / \mathrm{K} \\
\delta S_{b h s}=\frac{2 \pi}{\kappa c^{2}} K_{B H s} \delta R_{b h s}\end{array}$ \\
\hline 1 & $5 \mathrm{M}_{\odot}$ & 7375 & $2.483 \times 10^{63}$ \\
\hline 2 & $6 \mathrm{M}_{\odot}$ & 8850 & $3.575 \times 10^{63}$ \\
\hline 3 & $7 \mathrm{M}_{\odot}$ & 10325 & $4.867 \times 10^{63}$ \\
\hline 4 & $8 \mathrm{M}_{\odot}$ & 11800 & $6.357 \times 10^{63}$ \\
\hline 5 & $9 \mathrm{M}_{\odot}$ & 13275 & $8.045 \times 10^{63}$ \\
\hline 6 & $10 \mathrm{M}_{\odot}$ & 14750 & $9.932 \times 10^{63}$ \\
\hline 7 & $11 \mathrm{M}_{\odot}$ & 16225 & $12.018 \times 10^{63}$ \\
\hline 8 & $12 \mathrm{M}_{\odot}$ & 17700 & $14.303 \times 10^{63}$ \\
\hline 9 & $13 \mathrm{M}_{\odot}$ & 19175 & $16.786 \times 10^{63}$ \\
\hline 10 & $14 \mathrm{M}_{\odot}$ & 20650 & $19.468 \times 10^{63}$ \\
\hline 11 & $15 \mathrm{M}_{\odot}$ & 22125 & $22.349 \times 10^{63}$ \\
\hline 12 & $16 \mathrm{M}_{\odot}$ & 23600 & $25.428 \times 10^{63}$ \\
\hline 13 & $17 \mathrm{M}_{\odot}$ & 25075 & $28.706 \times 10^{63}$ \\
\hline 14 & $18 \mathrm{M}_{\odot}$ & 26550 & $32.182 \times 10^{63}$ \\
\hline 15 & $19 \mathrm{M}_{\odot}$ & 28025 & $35.857 \times 10^{63}$ \\
\hline 16 & $20 \mathrm{M}_{\odot}$ & 29500 & $39.731 \times 10^{63}$ \\
\hline
\end{tabular}

Table 2.

\begin{tabular}{|c|c|c|c|c|c|}
\hline \multicolumn{7}{|c|}{ Change in entropy of spinning black holes in AGN. } \\
\hline $\begin{array}{c}\text { S1. } \\
\text { No. }\end{array}$ & $\begin{array}{c}\text { Mass of } \\
\text { BHs (M) }\end{array}$ & $\begin{array}{c}R_{b h s}=1475\left(M / M_{\odot}\right) \\
\text { (in metre) }\end{array}$ & $\begin{array}{c}\log \left(\mathrm{R}_{\text {bhs }}\right) \\
\text { (in } \\
\text { metre) }\end{array}$ & $\begin{array}{c}\text { Entropy change of } \\
\text { black holes }\left(\delta S_{b h}\right) \text { in } \\
\mathrm{J} / \mathrm{K}\end{array}$ & $\log \left(\delta S_{b h}\right)$ \\
\hline 1 & $\begin{array}{c}1 \times 10^{6} \\
\mathrm{M}_{\odot}\end{array}$ & $1.475 \times 10^{9}$ & 9.1687 & $9.932 \times 10^{73}$ & 73.9970 \\
\hline 2 & $\begin{array}{c}2 \times 10^{6} \\
\mathrm{M}_{\odot}\end{array}$ & $2.950 \times 10^{9}$ & 9.4698 & $39.731 \times 10^{73}$ & 74.5991 \\
\hline
\end{tabular}




\begin{tabular}{|c|c|c|c|c|c|}
\hline 3 & $\begin{array}{c}3 \times 10^{6} \\
\mathrm{M}_{\odot}\end{array}$ & $4.425 \times 10^{9}$ & 9.6459 & $89.396 \times 10^{73}$ & 74.9513 \\
\hline 4 & $\begin{array}{c}4 \times 10^{6} \\
\mathrm{M}_{\odot}\end{array}$ & $5.900 \times 10^{9}$ & 9.7708 & $15.892 \times 10^{74}$ & 75.2012 \\
\hline 5 & $\begin{array}{c}5 \times 10^{6} \\
\mathrm{M}_{\odot}\end{array}$ & $7.375 \times 10^{9}$ & 9.8677 & $24.832 \times 10^{74}$ & 75.3948 \\
\hline 6 & $6 \times 10^{6} \mathrm{M}_{\odot}$ & $8.850 \times 10^{9}$ & 9.9469 & $35.758 \times 10^{74}$ & 75.5533 \\
\hline 7 & $\begin{array}{c}7 \times 10^{6} \\
\mathrm{M}_{\odot}\end{array}$ & $1.032 \times 10^{10}$ & 10.0136 & $48.671 \times 10^{74}$ & 75.6872 \\
\hline 8 & $\begin{array}{c}8 \times 10^{6} \\
\mathrm{M}_{\odot}\end{array}$ & $1.180 \times 10^{10}$ & 10.0718 & $63.570 \times 10^{74}$ & 75.8033 \\
\hline 9 & $\begin{array}{c}9 \times 10^{6} \\
\mathrm{M}_{\odot}\end{array}$ & $1.327 \times 10^{10}$ & 10.1228 & $80.456 \times 10^{74}$ & 75.9056 \\
\hline 10 & $\begin{array}{c}1 \times 10^{7} \\
\mathrm{M}_{\odot}\end{array}$ & $1.475 \times 10^{10}$ & 10.1687 & $9.932 \times 10^{75}$ & 75.9970 \\
\hline 11 & $\begin{array}{c}2 \times 10^{7} \\
\mathrm{M}_{\odot}\end{array}$ & $2.950 \times 10^{10}$ & 10.4698 & $39.731 \times 10^{75}$ & 76.5991 \\
\hline 12 & $\begin{array}{c}3 \times 10^{7} \\
\mathrm{M}_{\odot}\end{array}$ & $4.425 \times 10^{10}$ & 10.6459 & $89.396 \times 10^{75}$ & 76.9513 \\
\hline 13 & $\begin{array}{c}4 \times 10^{7} \\
\mathrm{M}_{\odot}\end{array}$ & $5.900 \times 10^{10}$ & 10.7708 & $15.892 \times 10^{76}$ & 77.2012 \\
\hline 14 & $\begin{array}{c}5 \times 10^{7} \\
\mathrm{M}_{\odot}\end{array}$ & $7.375 \times 10^{10}$ & 10.8677 & $24.832 \times 10^{76}$ & 77.3948 \\
\hline 15 & $6 \times 10^{7} \mathrm{M}_{\odot}$ & $8.850 \times 10^{10}$ & 10.9469 & $35.758 \times 10^{76}$ & 77.5533 \\
\hline 16 & $\begin{array}{c}7 \times 10^{7} \\
\mathrm{M}_{\odot}\end{array}$ & $1.032 \times 10^{11}$ & 11.0136 & $48.671 \times 10^{76}$ & 77.6872 \\
\hline 17 & $\begin{array}{c}8 \times 10^{7} \\
\mathrm{M}_{\odot}\end{array}$ & $1.180 \times 10^{11}$ & 11.0718 & $63.570 \times 10^{76}$ & 77.8033 \\
\hline 18 & $\begin{array}{c}9 \times 10^{7} \\
\mathrm{M}_{\odot} \\
\end{array}$ & $1.327 \times 10^{11}$ & 11.1228 & $80.456 \times 10^{76}$ & 77.9056 \\
\hline 19 & $\begin{array}{c}1 \times 10^{8} \\
\mathrm{M}_{\odot}\end{array}$ & $1.475 \times 10^{11}$ & 11.1687 & $9.932 \times 10^{77}$ & 77.9970 \\
\hline 20 & $\begin{array}{c}2 \times 10^{8} \\
\mathrm{M}_{\odot}\end{array}$ & $2.950 \times 10^{11}$ & 11.4698 & $39.731 \times 10^{77}$ & 78.5991 \\
\hline 21 & $\begin{array}{c}3 \times 10^{8} \\
\mathrm{M}_{\odot}\end{array}$ & $4.425 \times 10^{11}$ & 11.6459 & $89.396 \times 10^{77}$ & 78.9513 \\
\hline 22 & $\begin{array}{c}4 \times 10^{8} \\
\mathrm{M}_{\odot}\end{array}$ & $5.900 \times 10^{11}$ & 11.7708 & $15.892 \times 10^{78}$ & 79.2012 \\
\hline 23 & $\begin{array}{c}5 \times 10^{8} \\
\mathrm{M}_{\odot}\end{array}$ & $7.375 \times 10^{11}$ & 11.8677 & $24.832 \times 10^{78}$ & 79.3948 \\
\hline 24 & $\begin{array}{c}6 \times 10^{8} \\
\mathrm{M}_{\odot}\end{array}$ & $8.850 \times 10^{11}$ & 11.9469 & $35.758 \times 10^{78}$ & 79.5533 \\
\hline 25 & $\begin{array}{c}7 \times 10^{8} \\
\mathrm{M}_{\odot}\end{array}$ & $1.032 \times 10^{12}$ & 12.0136 & $48.671 \times 10^{78}$ & 79.6872 \\
\hline 26 & $\begin{array}{c}8 \times 10^{8} \\
\mathrm{M}_{\odot}\end{array}$ & $1.180 \times 10^{12}$ & 12.0718 & $63.570 \times 10^{78}$ & 79.8033 \\
\hline
\end{tabular}




\begin{tabular}{|c|c|c|c|c|c|}
\hline 27 & $\begin{array}{c}9 \times 10^{8} \\
\mathrm{M}_{\odot}\end{array}$ & $1.327 \times 10^{12}$ & 12.1228 & $80.456 \times 10^{78}$ & 79.9056 \\
\hline 28 & $\begin{array}{c}1 \times 10^{9} \\
\mathrm{M}_{\odot}\end{array}$ & $1.475 \times 10^{12}$ & 12.1687 & $9.932 \times 10^{79}$ & 79.9970 \\
\hline 29 & $\begin{array}{c}2 \times 10^{9} \\
\mathrm{M}_{\odot}\end{array}$ & $2.950 \times 10^{12}$ & 12.4698 & $39.731 \times 10^{79}$ & 80.5991 \\
\hline 30 & $\begin{array}{c}3 \times 10^{9} \\
\mathrm{M}_{\odot}\end{array}$ & $4.425 \times 10^{12}$ & 12.6459 & $89.396 \times 10^{79}$ & 80.9513 \\
\hline 31 & $\begin{array}{c}4 \times 10^{9} \\
\mathrm{M}_{\odot}\end{array}$ & $5.900 \times 10^{12}$ & 12.7708 & $15.892 \times 10^{80}$ & 81.2012 \\
\hline 32 & $\begin{array}{c}5 \times 10^{9} \\
\mathrm{M}_{\odot}\end{array}$ & $7.375 \times 10^{12}$ & 12.8677 & $28.432 \times 10^{80}$ & 81.3948 \\
\hline
\end{tabular}

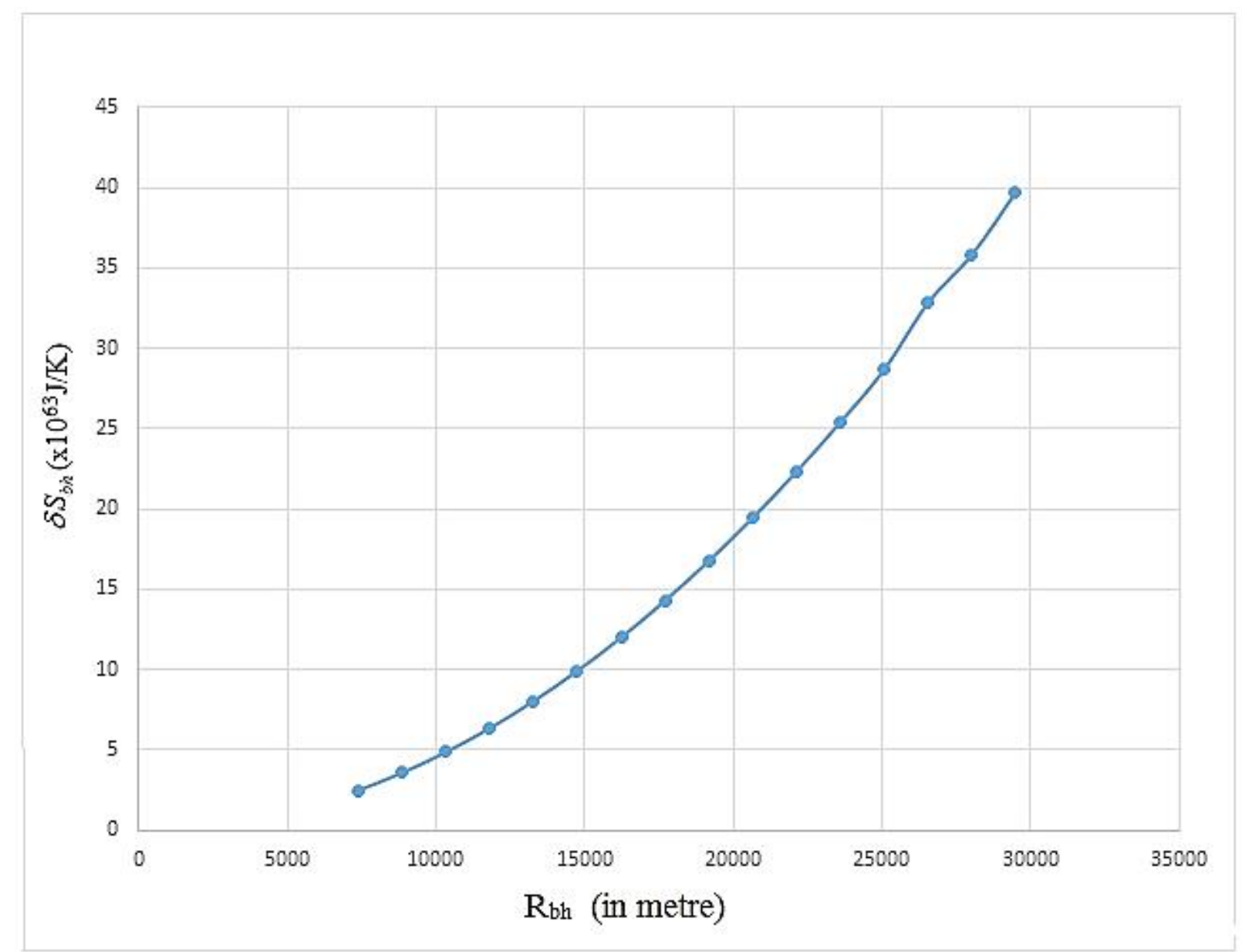

Fig. 1. The graph plotted between the radius of event horizon and corresponding change in entropy of the black hole in XRBs (Table 1). 


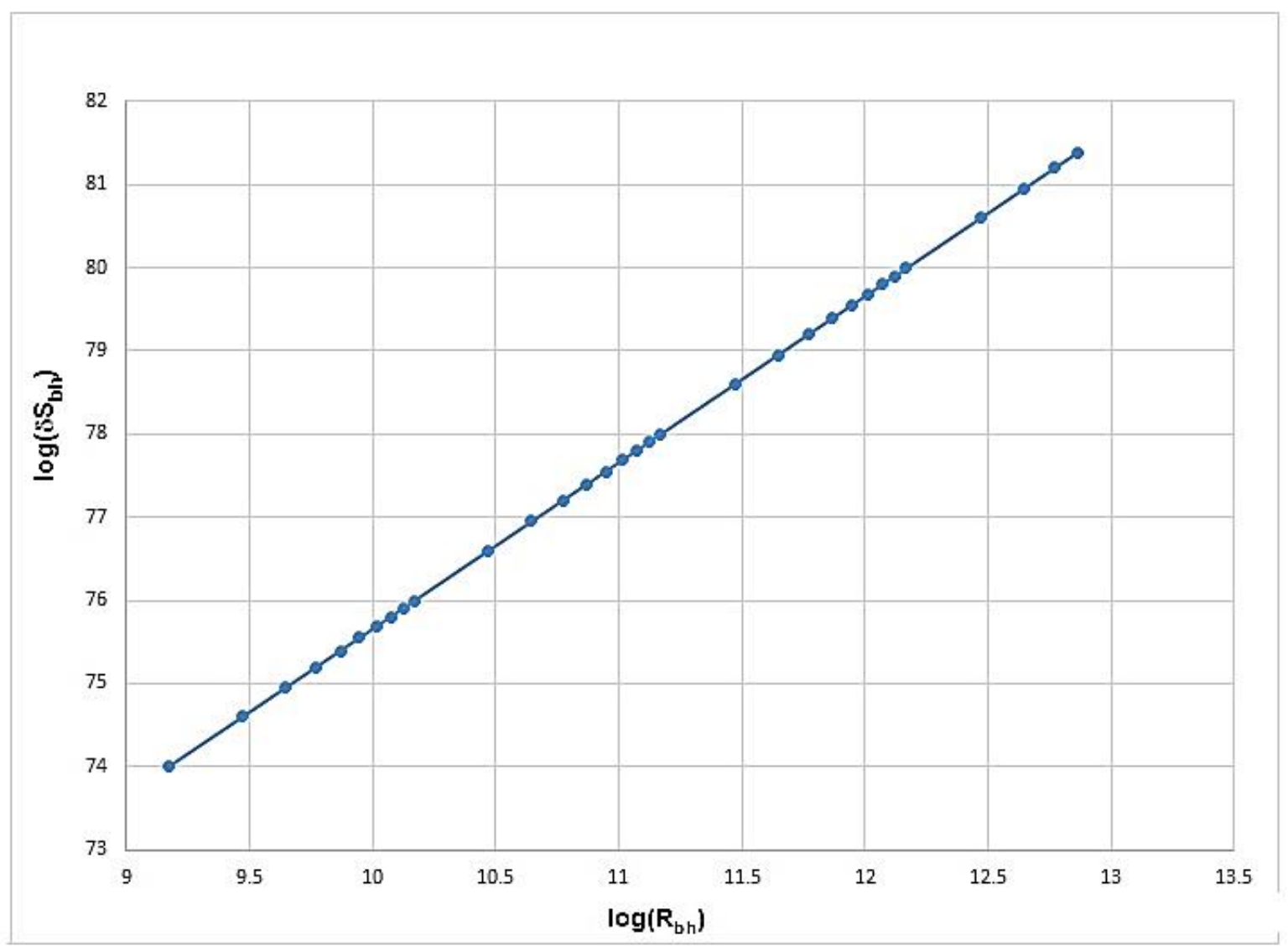

Fig. 2. The graph plotted bet ${ }^{\mathrm{n}}$ the radius of event horizon and corresponding change in entropy of the black hole in AGN (Table 2).

\section{DISCUSSION AND RESULT}

In present work, we have derived the formula for the change in entropy of spinning black holes $\delta S_{b h s}=\frac{2 \pi}{\kappa c^{2}} K_{B H s} \delta R_{b h s}$ using $E_{B H s}=K_{B H s} R_{s}^{\prime}$ in equation for change in entropy $\delta S_{b h}=\frac{2 \pi}{\kappa \cdot c^{2}} \delta E$ (Mahto et al. 2011b). We have also calculated the change in entropy of different test spinning black holes in X-ray binaries (XRBs) and Active Galactic Nuclei (AGN) and some remarkable results have been obtained. To know more about the results, graphs have been plotted between:

(i) the radius of event horizon $\left(R_{b h s}\right)$ of different test spinning black holes and their corresponding entropy change in XRBs (Fig. 1).

(ii) the radius of event horizon $\left(R_{b h s}\right)$ of different test spinning black holes and their corresponding entropy change in AGN (Fig. 2).

From the observation of the data in the Table 1 and 2, it is clear that the change in entropy of different test spinning black holes increases with increase in corresponding values of the mass/radius of the event horizon in both case of XRBs and AGN. From the observation 
of the graph in the Figure 1, it is clear that the change in entropy of different test black holes increases approximately linearly (slightly curved) with increase in corresponding values of the mass/radius of the event horizon in the case of XRBs, while exactly linear in the case of AGN. The graph plotted between the radius of event horizon $\left(R_{b h s}\right)$ of different test spinning black holes \& their corresponding change in entropy in XRBs (Fig. 1) has the same nature of the graph plotted between the radius of event horizon of different test black holes and their corresponding entropy in XRBs using the well known relation of entropy $S=A / 4=\pi R_{b h}^{2} / 4$ as proposed by Stephen Hawking (Hawking 1973, Mahto et al. 2012) and the graph plotted between the radius of event horizon of different test spinning black holes and their corresponding change in entropy in AGN (Fig. 2) is straight line justifying the validity of $E_{B H s}=K_{B H s} R_{b h s}$ as proposed by Mahto et al. (2011a), because the relation $\delta S_{b h s}=\frac{2 \pi}{\kappa c^{2}} K_{B H s} \delta R_{b h s}$ has been derived using relation $E_{B H s}=K_{B H s} R_{b h s}$. When we compared the present work with that of non-spinning black holes (Mahto et al. 2014), we see that the change in energy and entropy of spinning black holes are the exactly same to the non-spinning black holes, while the event horizon of spinning black holes is half to that of the non-spinning black holes of the same mass. This shows that the change in energy and entropy are mainly dependent on the mass and independent of the event horizon of black holes.

\section{CONCLUSIONS}

In the present research paper, we have drawn the following conclusions:

(i) The calculated values for change in entropy show that $\delta S \geq 0$ for each spinning black holes in XRBs and AGN. This result is good agreement with the second law of thermodynamics.

(ii) Larger the mass/ radius of event horizon, greater is the change in entropy of spinning black holes and vice-versa.

(iii) Our result has good agreement in XRBs with relation of entropy $S=A / 4=\pi R_{b h}^{2} / 4$ as proposed by Hawking.

(iv) Mahto model for energy of the spinning black hole is justified.

(v) The change in energy and entropy are mainly dependent on the mass and independent of the event horizon of black holes.

\section{ACKNOWLEDGEMENT}

The authors are obliged to Dr. Gopi Kant Jha, Former Head \& Prof. of Physics, L.N.M.U Darbhanga and Dr. Prof. M.S.H. John, Principal, Marwari College Bhagalpur for their inspiration and motivation. 


\section{References}

[1] Wald R. M., The thermodynamics of black holes. Living reviews in relativity (2001).

[2] Bardeen J. M., Carter B., Hawking S. W., Commun. Math. Phy. 31(2) (1973) 161-170.

[3] Mahto D., Kumari K., Sah R. K., Prakash V., Bulletin of Pure and Applied Sciences 30D(1) (2011) 157-162.

[4] Kumari K., Mahto D., Chandra G., Sah R. K., Singh K. M., Bulletin of Pure and Applied Sciences 29D(2)(2010) 183-187.

[5] Mahto D., Kumari K., Sah R. K., Singh K. M., Astrophysics and Space Science 337(2) (2012) 685-691.

[6] Dipo Mahto, Tanveer Ali Khan, Ashok Kumar, Raj Kumar Sah, International Letters of Chemistry, Physics and Astronomy 4 (2014) 12-19

[7] D. Mahto, R. N. Mehta, K. Parhi, K. M. Singh, R. K. Sah, Journal of Physical Science $3(2)(2013) 1-5$.

[8] Einstein A., Annals of Mathematics (Second Series) 40(4) (1939) 922-936.

[9] Transchen J., An introduction to black hole evaporation, General Relativity and Quantum Cosmology (2000), arXiv: gr- qc/0010055V1.

[10] Dabholkar A., Current Science 89(12) (2005) 2054-2063.

[11] Mahto D., Prakash V., Prasad U., Singh B. K., Singh K. M., Astrophysics Space Science 343(1) (2013) 153-159.

[12] R. Narayan, New Journal Physics 7(1) (2005) 1-31.

[13] D. Mahto, M. S. Nadeem, M. Ram and K. Vineeta, Journal of Gravity, 2013, Article ID: 222676.

[14] Ferrarese L., Ford H., Space Science Reviews 116 (2005) 523-624.

[15] Madejski G., Black Holes in Active Galactic Nuclei, SLAC-PUB-9702, March 2003.

[16] R. D. Blandford, Astrophysics and Space Science 261 (1999) 245-252.

[17] Mahto D., Mehta R. N., Prasad U., Sah R. K., Singh, K. M., International Journal of Astrophysics and Space Science 1(4) (2013) 52-55. 